\title{
23 De-Zentrierungen und Ex-Zentrierungen. Die quasi-transzendentalen Unternehmungen von Heidegger und Plessner heute
}

Kants kopernikanische Revolution ist - gegen seine Intention - immer wieder nachgeahmt worden, wirkungsmächtig verschoben und versetzt nicht zuletzt von Martin Heidegger. Sie wurde zu einem Modell für die Dezentrierung von Zentrismen, das attraktiv schien, denn wer möchte nicht zentristische Standpunkte überwinden? Aber dieses Avantgarde-Modell hat sich in praxi wider auch seine besten Intentionen verkehrt. Statt der Dezentrierung trat die dualistische Aushebelung der bisherigen Lebensmitte ein, an deren Funktionsstelle die beabsichtigte Dezentrierung als das neue Zentrum geriet. Zudem paralysieren sich zu viele Kopernikanische Revolutionen gegenseitig und überlassen am Ende dem Dezisionismus das Feld. Gegen diese westlich-moderne Tendenz zum Abbau einer in der Mitwelt geteilten Lebensmitte, die das geistige Niveau von Personalität hält, hatte Max Scheler die Ekstasis entworfen, d. h. eine Vereinigung von Leben und Geist, deren ontisch-ontologische Differenz nicht aufgehoben werden kann. Sie wurde, im Kontrast zu Edmund Husserls Rückkehr in die klassisch dualistische Transzendentalphilosophie, in zwei quasi transzendentalen Unternehmungen entfaltet, einerseits durch Heidegger in existenzialund seinsphilosophischer Richtung und andererseits durch Helmuth Plessner in Richtung auf eine Philosophische Anthropologie.

Die Transformation von Schelers Ekstasis in Plessners und Heideggers Philosophien fehlt in der heutigen systematischen Diskussion. Hermann Schmitz und Peter Sloterdijk halten - gegen Heideggers offizielles Selbstverständnis Heideggers Schaffen teilweise für zugehörig zu der Philosophischen Anthropologie, die Scheler und Plessner begründet haben. Giorgio Agamben und Jacques Derrida, die beide von Heidegger inspiriert wurden und sein Vorurteil gegen die Philosophische Anthropologie teilen, kritisieren Heideggers einschlägige Schriften als noch immer eine Anthropozentrik, von der sie befreien könnten. Aber wie soll diese erneute Geste der Überwindung einer Zentrik gelingen: durch eine neuerliche Dezentrierung des Humanismus, wie schon bei Heidegger, oder durch eine exzentrische Anhebung der Mitte personaler Lebensformen für ihre gemeinsame Zukunft? Plessners Exzentrierung ist keine dualistische Abspaltung vom Zentrum, die sich in ein neues Zentrum verwandeln kann, sondern eine geistig-kulturelle Anhebung der Lebensmitte in personalen Lebensformen.

Im Folgenden möchte ich zu dieser Diskussion aus Sicht der Plessnerschen Philosophischen Anthropologie einen Beitrag leisten, indem ich Schelers Anre- 
gungspotential rekonstruiere, die Frage der Humanismus-Kritik umstelle und dafür ein nötiges Minimum an Ermöglichungsstrukturen von Welt (naturphilosophisch) und geschichtlicher Zukunft (geschichtsphilosophisch) in Erinnerung rufe. Diese Art und Weise von Weltlichkeit ermöglicht eine exzentrierende Anhebung der personalen Lebensmitte. Sie ging aber in Heideggers Einebnung der Differenz zwischen Welt und Umwelt verloren, ohne dass Agamben und Derrida diesen Weltverlust als Problem ansehen können. Beide unterscheiden nicht wie Plessner zwischen drei „Achsendrehungen“, d. h. nicht zwischen erstens der Entstehung der Achsenkulturen einschließlich Christentum, zweitens der Verweltlichung des Christentums zur westlichen Moderne einschließlich ihres $\mathrm{Hu}$ manismus und drittens der Überwindung des Dezisionismus in einer Vielfalt auch außerwestlicher Modernen. Nicht der Humanismus, der Dezisionismus ist das Hauptproblem in der westlichen Moderne und ihrer Globalisierung. Die von Agamben und Derrida ignorierte Kritik Plessners an Heideggers Anthropozentrik war nicht nur früher, sondern auch besser als ihre neuerlichen Versuche, Heideggers Überbietung zu überbieten.

\subsection{Die Abkopplung der transzendentalen Ermöglichungsfrage von ihrer klassischen Beantwortung durch das Selbstbewusstsein: Heidegger und Plessner antworten auf Schelers Ekstasis von Geist und Leben quasi transzendental}

Die Spezifik der modernen Philosophie ist häufig nach dem Modell der Kantischen Transzendentalphilosophie in Analogie mit einer kopernikanischen Revolution aufgefasst worden. Dem entsprechend hat dann Philosophie die Frage nach den Ermöglichungsbedingungen der wissenschaftlichen Erfahrung zu stellen. Während das auf den Gegenstand gerichtete Bewusstsein in der Erfahrungswissenschaft mit der Bestimmung seiner Inhalte beschäftigt ist, wird dieses Bewusstsein in der Philosophie vom Standpunkt des Selbstbewusstseins aus auf seine Ermöglichungsbedingungen hin beobachtet. Das Gegenstandsbewusstsein tritt nicht voraussetzungslos an die Empirie seiner Inhalte heran, sondern durch Anschauungs- und Denkformen vermittelt. Das auf Gegenstände zentrierte Erfahrungsbewusstsein wird so vom Selbstbewusstsein her dezentriert. Philosophie als Wissenschaft lasse sich nicht mehr auf die Annahme gründen, ,alle unsere Erkenntnis müsse sich nach den Gegenständen richten“, 
sondern erfordere eine Art von kopernikanischer Wendung in die Annahme, „die Gegenstände müssen sich nach unserem Erkenntnis richten“:

Es ist hiermit ebenso, als mit den ersten Gedanken des Copernicus bewandt, der, nachdem es mit der Erklärung der Himmelsbewegungen nicht gut fortwollte, wenn er annahm, das ganze Sternheer drehe sich um den Zuschauer, versuchte, ob es nicht besser gelingen möchte, wenn er den Zuschauer sich drehen und dagegen die Sterne in Ruhe ließ. (Kant 1971, 22f.)

Dasjenige an Struktur- und Funktionsbedingungen, welches Erfahrungswissenschaft so ermöglicht, unterliegt nicht eben dieser erfahrungswissenschaftlichen Bestimmung von gegenstandsbezogenen Inhalten. Die in der Ermöglichungsfrage gewonnene Freiheit zur Vernunft hat sich in ihrer Verantwortung für die Folgen ihres Gebrauchs zu bewähren. Über die theoretische Aufgabe, die Erkenntnisvermögen gegen ihren dogmatischen Gebrauch $\mathrm{zu}$ begrenzen und in Bezug aufeinander in eine richtige Proportion zu bringen, hinausgehend, besteht das kritische Geschäft der Transzendentalphilosophie vor allem darin, die moralische Praxis im Sinne einer Selbstgesetzgebung der Vernunft zu begründen.

Selbst Edmund Husserl, der ursprünglich in seinen Logischen Untersuchungen noch kein transzendentalphilosophisches Selbstverständnis hatte, ist ab seinen Ideen bei allen Variationen und Modifikationen doch auf dieses Grundmuster im Philosophieren, eine als absolut geltende Subjektivität als letzten Ermöglichungsgrund, zurückgekommen. Seine Wiederholung der Transzendentalphilosophie radikalisiert sie sogar im Sinne der Apodiktizität der eigenwesentlichen Reinheit von Intentionalität (Descartes) für den absolut uninteressierten Betrachter (Husserl 1992, 247, 260).

In der wirklich sich selbst verstehenden universalen Epoché zeigt es sich, dass es für die Seelen in ihrer Eigenwesentlichkeit überhaupt keine Trennung des Außereinander gibt. Was in der natürlich-mundanen Einstellung des Weltlebens vor der Epoché ein Außereinander ist, durch Lokalisation der Seelen an den Leibern, das verwandelt sich in der Epoché in ein reines intentionales Ineinander. Damit verwandelt sich die Welt, die schlicht seiende, und in ihr die seiende Natur, in das allgemeinschaftliche Phänomen ,Welt', ,Welt für alle wirklichen und möglichen Subjekte', von denen keines sich der intentionalen Implikation entziehen kann, der gemäß es in den Horizont eines jeden Subjekts vorweg hineingehört. (Ebd., 259)

Schaut man sich die systematische Problemgeschichte dieses Philosophieverständnisses in der ersten Hälfte des 20. Jahrhunderts an, so gab es viele Umstellungen und Erweiterungen der transzendentalen Verfahrensweise schon unter den Husserl-Schülern. Ich möchte hier an die Wegegabelung durch Max 
Schelers Kritik an Husserl erinnern, weil sie dann $\mathrm{zu}$ den beiden nur noch quasi-transzendentalen Unternehmungen von Martin Heidegger und Helmuth Plessner seit den 1920er Jahren geführt hat. Scheler hat, wenngleich noch im Gewande einer Geistesmetaphysik, eine methodisch revolutionäre Umstellung in der Phänomenologie vorgenommen. In seiner sog. Neutralisierung gegen Cartesianische Dualismen befreit er das Philosophieren von der Dichotomie, dass sich die Phänomene der Erwartung fügen müssten, entweder nur res extensa oder nur res cogitans sein zu können. Umgekehrt: Phänomene, die sich sowohl als res extensa (Physisches) als auch als res cogitans (Psychisches) verhalten, kandidieren als solche, die leben. Seine Kategorien verhalten sich gegenüber den Dichotomien als ,indifferent“ bzw. „neutral“, um neue Unterscheidungen von einem jeweils Dritten im Leben her, das nicht mehr reflexiver Zuschauer oder nur Beobachter, sondern Teilnehmer ist, zu ermöglichen. Scheler problematisiert die moderne Fehlidentifikation des Gegensatzes, entweder außen oder innen, mit den Gegensätzen, entweder physisch oder psychisch, und, entweder anders oder eigen. Aus dieser Fehlidentifikation erwächst das im modernen Dualismus unlösbare Problem, wie man vom eigenen Ich, das im Inneren als Psychisches gegeben sei, zum fremden Ich, das aber doch im Äußeren nur physisch gegeben sei, gelangen könne, vermeintlich durch Einfühlung in den Anderen und Analogieschluss aus dem eigenen auf das fremde Ich (siehe Scheler 1985, 209-258).

Stattdessen stellt Scheler die Frage dahingehend um, wie Lebewesen von den sinngemäßen „Ganzheitsstrukturen“ in ihrem Ausdrucksverhalten ausgehend dazu kommen, sinnliche Erscheinungen ,als Fundamente dieser Strukturen fungieren“ zu lassen und sie „Zeichen- und Darstellungsfunktionen für diese Ganzheiten übernehmen können“ (Scheler 1985, 258). Mit dieser Umstellung wird auch die Differenz zwischen dem Ontischen und dem Ontologischen neu verstanden. Wenn Leben einen „ontisch einheitlichen Lebensprozess“ bildet, dann darf es auch ontologisch keiner dualistischen Zugangsweise geopfert werden. Es ist nicht entweder nur physiologisch oder nur psychologisch zu verstehen:

Endlich müssen nach unserer Lehre auch die geistigen Akte, da und insofern sie ihre ganze Tätigkeitsenergie aus der lebendigen Triebsphäre beziehen und ohne irgendeine ,Energie‘ sich für unsere Erfahrung, auch für die eigene, nicht manifestieren können, stets ein physiologisches und psychisches Parallelglied besitzen. (Scheler 1995, 77f., siehe auch 74)

Diese Thematisierung von Leben wirft das neue Problem auf, wie sich überhaupt noch Lebensformen von Lebensformen unterscheiden lassen, die alle in sich bereits nach alter, primär erkenntnistheoretischer Terminologie Subjekt- 
Objekte und Objekt-Subjekte darstellen, eine aus dem deutschen Idealismus bekannte Figur. Um hier noch Differenzierungen zu ermöglichen, führt Scheler unter den Dritten, die aus den Dichotomien des Entweder-Oder der westlichen Moderne herausführen, Figuren der Liebe und damit des verlebendigten Geistes oder des vergeistigten Lebens ein. Liebesbewegungen sind - gegenüber dem „existential-empirischen Sein“ und gegenüber dem „Seinsollen“ - ein

[...] Drittes, gegen diesen Unterschied noch Indifferentes: dasselbe ,Sein` z. B., das in dem Satze liegt: ,Werde der du bist', der eben etwas anderes besagt als ,du sollst so und so sein', aber auch etwas anderes als ,existentiales empirisches Sein'. Denn was man in diesem letzteren Sinne ,ist', braucht man nicht zu werden. $\left(\right.$ Scheler 1985, 162) ${ }^{1}$

Unter diesen Figuren eines Dritten hat die der Ekstasis, des Aus-sich-Heraus ins Andere Hinein-Versetztseins, eine besondere wirkungsgeschichtliche Rolle erhalten. An sie als eleusisches Mysterium erinnert Diotima in Platons Symposium, wo Eros aus der leiblichen zur höchsten Liebe hinaufführt. Die Ekstasis taucht in der Formierungsphase der Agape im Christentum bei Philòn (Philo Alexandrinus oder Philo Judaeus) gleich in den ersten Jahrzehnten nach Jesus' Tod erneut auf. Dieser jüdische Gelehrte interpretierte die Heiligen Schriften des Judentums und die antik griechische Philosophie in Bezug aufeinander selbst in altgriechischer Sprache. So entsteht eine Neuinterpretation der Ekstasis:

Wen diese begeisterte Erhebung zu Gott, die göttliche Ekstase ergriffen hat, in dem ist die Sonne des eigenen Bewusstseins untergegangen vor dem Aufgange des göttlichen Lichtes. Es ist ihm zu Mute, wie einem sprach- und bewusstlosen Kinde; der göttliche Geist wohnt in ihm und bewegt ihn willenlos, wie die Saiten eines musikalischen Instrumentes. (Noack 1897, 672) ${ }^{2}$

Scheler setzt Plotins Fassung (3. Jh.) voraus und nimmt die Ekstasis aus der christlichen Mystik am gelebten Beispiel des Franz von Asissi (13. Jh. nach Christus) auf.

Es handelt sich um eine einzigartige Bewegung von ,Eros‘ und ,Agape‘ (einer in den Amor Dei und Amor in Deo ganz tief eingesenkten Agape) in einer urtümlich heiligen und genialischen Seele - und schließlich um eine Art so restloser Durchdringung beider, die das

1 Siehe zu den Sinngesetzen des emotionalen Lebens als einer universellen Grammatik im Ausdrucksverhalten Scheler 1985, 10, 22, 112, 210, 233-235. Zu Schelers problemgeschichtlicher Stellung Schloßberger 2005.

2 Artikel „Philòn“ in: Noack 1879, 672. 
größte und erhabenste Beispiel gleichzeitiger ,Vergeistigung des Lebens‘ und ,Verlebendigung des Geistes ' darstellt, das mir bekannt geworden ist. (Scheler 1985, 103) ${ }^{3}$

Diese Heraushebung des Vereinigungsproblems von Leben und Geist erfolgt im Kontext des Vergleichs der Achsenkulturen, d. h. nicht nur der drei abrahamitischen Weltreligionen, sondern auch des Buddhismus und des Alten Persiens. Zudem hofft Scheler gegen Nietzsche zeigen zu können, dass die Agape nicht auf einem Ressentiment beruhen muss, wenngleich sie so verzerrt werden kann (Scheler 1972, 80-95). Unabhängig von der Stichhaltigkeit dieses Versuchs ist damit aber auch klar, dass sich Scheler gegen Religionen und Ideologien wendet, insofern sie Ressentiments kultivieren, also Askese für ein schwaches statt starkes Leben (Nietzsche) in Dienst nehmen. Gleichwohl bleibt für Scheler - wie für jeden an Gott Gläubigen - der letzte Seinsgrund (ens a se) nicht innerhalb des Lebens, sondern nur „außerhalb alles dessen, was wir ,Leben“ im weitesten Sinne nennen können“ (Scheler 1995, 37).

Diese einerseits methodisch und thematisch für die Vereinigung von Leben und Geist revolutionäre Öffnung und jene andererseits systematische Konservierung des Geistes außerhalb vom Leben in Schelers Werk, das an seinem frühen Ende auf eine Philosophische Anthropologie zuläuft, befriedigte die nachdenklichen Zeitgenossen nicht. Ernst Cassirer, der in Davos 1929 mit Heidegger über Philosophische Anthropologie disputiert hat, hat die Aufgabe, die allgemein der Philosophie aus Schelers Arbeiten nach dessen plötzlichen Tod 1928 erwächst, treffend wie folgt formuliert:

Der Geist braucht nicht mehr als ein allem Leben fremdes oder feindliches Prinzip betrachtet, sondern er kann als eine Wendung und Umkehr des Lebens selbst verstanden werden - eine Wandlung, die es in sich selbst erfährt, in dem Maße, als es aus dem Kreise des bloß organischen Bildens und Gestaltens in den Kreis der ,Form', der ideellen Gestaltung, eintritt. (Cassirer 1993, 52f.)

Aber Cassirers eigener Lösungsvorschlag, rückwirkend die von ihm zuvor entwickelten symbolischen Formen zwischen Wirklichkeit und Menschen zu schieben, um so die Wandlung funktional zu erklären (ebd., 51), war nicht konkurrenzlos. Heidegger und Plessner hatten bereits seit dem Beginn der 1920er Jahre genauestens mit Schelers Werk gearbeitet.

3 Vgl. zu Franz von Assisi Scheler 1985, 97-102, zu den beiden bei ihm vereinigten, ansonsten entgegen gesetzten Polen der „kosmisch-vitalen Einsfühlung“ und der „akosmistischen Personliebe“ siehe ebd., 137. 
Schelers Problemkonstellation, die Vereinigung von Leben und Geist nach Darwins Evolutionstheorie und Nietzsches Kritik an der Geistesmetaphysik neu denken zu können, führte einerseits in Heideggers frühe, Aristoteles neu interpretierende Hermeneutik der Faktizität und in seine sich anschließende Existenz- und Seinsphilosophie hinein, und anderseits in Helmuth Plessners Philosophische Anthropologie. Bei beiden Autoren gibt es eine inhaltliche Vervielfältigung der Ekstasis von Scheler in dem folgenden formalen Sinne: Etwas führt aus sich heraus in etwas Anderes hinein, ohne sich im Rückbezug aus dem Anderen in der Identifikation mit sich selbst beruhigen $\mathrm{zu}$ können und auch ohne einfach vom Anderen aufgesogen werden zu können. Etwas wird im Anderen inhomogen und bleibt dies auch noch im Rückbezug, der keine höhere Homogenität wird, die im Hegelschen Sinne aufheben könnte. In der Ekstasis wird jemand von sich fortgerissen ins Andere und kann sich doch auch nicht dem Anderen auf Dauer assimilieren. Der letzte Seinsgrund ist so keine substanziell in sich ruhende Identität mehr, sondern bleibt differentiell in der Bewegungsdynamik einer Beziehung, die mehr ist als ihre Glieder ohne sie sein können.

Hier bahnt sich methodisch, nicht metaphysisch gesehen an, was Niklas Luhmann später im Rückblick die Umstellung von der klassisch idealistischen Identität aus Differenz und Identität auf eine Differenz aus Identität und Differenz genannt hat (Luhmann 1984, 26f., 600f., 606f.). Das Primat in der Integration von Differenz und Identität wandert von der Identität in die Differenz. Man könnte auch - Heidegger und Plessner angemessener - formulieren, die Fragen öffnen sich aus ihren Antworten heraus in eine erneute Fraglichkeit. Die Antwortlichkeit, etwa im Sinne von bekannter Verantwortlichkeit, dominiert nicht mehr die Fraglichkeit, die sich durch das Medium ihrer geschichtlichen Antworten wiederherstellt. Und in den Formen der Zeitlichkeit, die Heidegger ausdrücklich Ekstasen nennt, gesprochen: Es zeichnet sich durch diese Tendenz $\mathrm{ab}$, wie wenig die Zukünftigkeit nur eine Wiederholung der Vergangenheit sein kann. Die Differenz zur Wiederholung der Vergangenheit wird größer, unsicherer, ein Wagnis, ein offener Ausgang, ein Wunder, ein Ereignis, wie immer die Terminologie ausfällt (siehe Krüger 2006b u. 2006c).

Gewiss sind die Foci in Plessners und Heideggers Philosophien von entgegen gesetzter Anordnung, weshalb Heidegger und Plessner zu Recht als die Gegenspieler wahrgenommen wurden, ob von Georg Misch, Nicolai Hartmann (Kalckreuth 2019), Ernst Cassirer, Josef König und anderen Zeitgenossen (Krüger 2001, 2009a, 2016c). Die Zentrierung aufs Dasein (Zuhandenheit, Vorhandenheit) wird bei Heidegger durch die Erschließung der Existenzialität des Daseins aus seinem Seinsbezug dezentriert (Heidegger 1993). Der Seinsbezug des Daseins gewinnt in seiner Spätphilosophie noch eine Freiheit gegenüber der indi- 
viduellen Existenzialität. Auf der Seite Plessners gibt es in der Naturphilosophie eine dreifache Exzentrierung, zunächst der anorganischen Dinge im Leben (Positionalität), dann des azentrischen Lebens im zentrischen Leben (zentrische Positionalität) und schließlich die Erschließung personalen Lebens aus der Exzentrierung des zentrischen Lebens (exzentrische Positionalität; Plessner 1975). Es werden auch die orientierenden Leistungen beider Philosophien einander entgegengesetzt bleiben. Heidegger markiert zunächst klar seine Verwendung aller Unterscheidungen zugunsten der Existenzialität, dann in seiner Rektoratsrede (1934) in der Gemeinschaft und im Spätwerk der Seinsphilosophie wandert die Markierung gar ins Sein ab, wo früher Gott einmal war. Bei Plessner hält sich eine aisthetische Markierung der leiblichen Mitwelt für die Angabe der Seite, von der her unterschieden wird, durch. Er hat sie exemplarisch im Lachen und Weinen herausgehoben (Plessner 1982f). Sie betrifft den Grenzübergang zwischen den sinngemäßen Bewegungsrichtungen von Leibesausdrücken und den Geistesformen, die nicht lokalisiert und temporalisiert werden können.

Aber bei allen Entgegensetzungen zwischen Plessner und Heidegger fallen doch auch die strukturellen Parallelen in der Anlage dieser Unternehmungen auf. Schelers Ekstasis wuchert sich aus den Aufhebungen der Hegelschen Dialektik in versöhnliche Enden der Weltgeschichte in beiden Werken heraus. Die Heraussetzung aus sich ins Andere und Fremde ist so heikel und prekär, dass der Rückweg zu sich nicht mehr gesichert werden kann. Die künftige Aneignung der gewesenen Selbstentfremdung ist im Geiste (Hegel) nicht mehr sicher, weder dem Wissen noch dem Glauben nach. Die Reproduktion der Lebenswelt geht in ihrer wissenschaftlich-technischen Realisierung als Krisis (Husserl) unter. Nicht dass es Dezentrierungen vom Anderen als dem Vertrauten her gibt, ist unter Heidegger und Plessner strittig, hier des Daseins in die Existenzialität und der Existenzialität nochmals ins Sein als Sinn, dort des Anorganischen im Leben, des a-zentrischen durch das zentrische Leben und schließlich dieser zentrischen nochmals durch eine exzentrische Positionalität. Es ist auch nicht strittig zwischen Plessner und Heidegger, dass es sich bei den Dezentrierungsbewegungen um sich selbst zeitigende, in die prinzipiell offene Zukunft verweisende Ermöglichungsstrukturen handelt, die sich vom ahistorischen transzendentalen Selbstbewusstsein befreien. Beide setzen Diltheys Historisierung der Kantschen Vernunft voraus. Die transzendentale Frage nach den funktionalen Ermöglichungsstrukturen gilt nicht mehr nur der wissenschaftlichen Erfahrung, sondern auch der Lebens- und Existenzerfahrung im weitesten Sinne. Sie wird ohne Rücksicht auf ihre traditionell oder klassisch transzendentale Antwort in einem Selbstbewusstsein quasi transzendental ausgeweitet. Sinnsein fundiert 
Existenzialität, die Dasein ermöglicht, von dem wir im Alltag ganz selbstverständlich ausgehen. Exzentrische Positionalität fundiert die Differenz zwischen a-zentrischen, zentrischen und de-zentrischen Lebensformen, die schließlich Dinge und Gestalten ermöglichen, die uns im Alltag so selbstverständlich vorkommen. ${ }^{4}$ Aber umso verschiedener werden von Heidegger und Plessner die Genese der Dezentrierungen und die Rückbezüge aus den Dezentrierungen heraus im Vollzug verstanden und erschlossen. Die phänomenologischen und hermeneutischen Methoden werden anders gehandhabt, die geschichtlichen Krisen der Zeitlichkeit anders aufgerollt, die quasi-transzendentalen Rückschlüsse aufs Ganze stellen einmal mehr existenziell-appellierende, das andere Mal mehr lebenspraktisch-therapeutische Aufgaben. Während für Heidegger die Existenz Leben fundiert, fundiert für Plessner Leben die Existenz (Plessner 1975, XIII).

\subsection{Der aktuelle Streit über Heideggers philosophische Anthropologie fordert den Vergleich mit Plessners Philosophischer Anthropologie heraus: Agamben, Derrida, Schmitz, Sloterdijk}

Für all jene philosophischen Diskurse der Gegenwart, in denen Heideggers Textkorpus als die entscheidende Autorität gilt, führt seine Wintervorlesung 1929-30 zu den ersten Schritten einer Wiederentdeckung der genannten Problemkonstellation zwischen ihm, Plessner und Scheler. Heidegger hat sich ausführlich in dieser Vorlesung Die Grundbegriffe der Metaphysik: Welt - Endlichkeit - Einsamkeit (Heidegger 1983) mit der Philosophischen Anthropologie auseinandergesetzt, mit der von Scheler explizit, mit der von Plessner implizit. Hermann Schmitz hatte schon 1996 in seinem Buch Husserl und Heidegger gezeigt, dass Heidegger nach Sein und Zeit 1927-29 in eine Transzendenzphase gerät, in der er sich deutlich aus Plessners exzentrischer Positionalität bedient, so auch in der Davoser Disputation mit Cassirer (Schmitz 1996, 374-377, 389, 408-411, 423, 471). Schmitz‘ Diagnose wurde jüngst von Großheim umfänglich bestätigt (Großheim 2018). Peter Sloterdijk ist weiter als Schmitz gegangen. Er hat in seinem Buch Nicht gerettet. Versuche nach Heidegger Heidegger gar zum philosophischen und historischen Anthropologen geadelt (Sloterdijk 2001, 153f., 156-159, 162, 173), was natürlich Heideggers Selbstverständnis vollkom-

4 Siehe zu den quasi transzendentalen Weisen zu philosophieren in Auseinandersetzung mit M. Foucault, J. Derrida und J. Austin Krüger 2001, 30f., 44, 48, 88f., 92f., 144f., 289f., 336f.; Krüger 2006a. 
men widerspricht. Heidegger hatte die Philosophische Anthropologie mehrfach als „Regionalontologie“ (Heidegger 1993, 45, 131) abgewertet, im Unterschied zu seiner eigenen Fundamentalontologie, und in der folgenden Anthropozentrik als Philosophie der Subjektivität karikiert: „Und gilt der Mensch als dasjenige Seiende, das in der Ordnung der Begründung einer absolut gewissen Erkenntnis das schlechthin Erstgegebene und Gewisseste ist, dann muss der so geplante Aufbau der Philosophie die menschliche Subjektivität in den zentralen Ansatz bringen“ (Heidegger 1975, 211). Diese Einschätzung der philosophischen Anthropologie in Heideggers Buch Kant und das Problem der Metaphysik enthielt 1929, als es mit der Widmung „Max Scheler zum Gedächtnis“ erschien, eine bewusste Fehlinformation, die in keiner Weise Schelers Die Stellung des Menschen im Kosmos und Plessners Die Stufen des Organischen und der Mensch (beide waren 1928 erschienen) entsprach.

Jacques Derrida und Giorgio Agamben haben der genannten Heideggerschen Wintervorlesung 1929/30 wichtige Interpretationen gewidmet, um ihre eigenen Philosophien in Übereinstimmung mit und Differenz zu Heidegger darzustellen. Derrida behauptet in Das Tier, das ich also bin sogar, Heideggers Vorlesung erlaube es ihm zu behaupten, dass sich seine Philosophie, die Derridas, schon immer um das Differieren der Lebensdifferenzen gedreht hätte (Derrida 2010, 62, 65). Über diese späte Aufpfropfung auf das Lebensthema kann man sich nur wundern. Und Agamben ist in Das Offene. Der Mensch und das Tier (2003) davon überzeugt, dass sich eben in dieser Vorlesung Heideggers noch seine Anthropozentrik zeige. Agamben möchte die Entkopplung von Körper und Seele radikalisieren, statt beide aus ihrer Trennung im Abendlande zu vereinigen (Agamben 2003, 84-88). Wir haben es schon in der Ankündigung sowohl bei Derrida als auch bei Agamben mit Überbietungen zu tun, die die Überbietungen Heideggers nochmals überbieten sollen.

Sowohl Derrida als auch Agamben sind mit der noch zu großen Anthropozentrik von Heidegger unzufrieden. Sie zeige sich in seiner Unterscheidung der weltlosen Steine von den weltarmen Tieren und den weltbildenden Menschen (Heidegger 1983, 263). Heideggers Umstellung der Frage nach der Spezifik des Menschen in die nach der Grundstimmung tiefer Langeweile gelinge nicht, weil sie die alten Dichotomien zwischen Schlaf, Unbewusstem, Bewusstem und Selbstbewusstem nicht überwinde. Derrida und Agamben plädieren für einen ethisch motivierten Ausweg aus der Ausbeutung des Tieres und schlagen das inzwischen übliche Verfahren der politischen Korrektheit vor: Der eine scharfe Gegensatz zwischen „dem“ Tier und „dem“ Menschen soll vervielfältigt werden in neue Grenzmarkierungen hinein, die auf den Bedeutungshof dessen, was Tiere entbehren und wessen sie beraubt seien, verzichten, so Derridas Vor- 
schlag in seinem l'animot (Derida 2010, 79f.). Man kennt Verfahren der Differenzierung aus der Vervielfältigung und Normalisierung von Geschlechtern, Klassen und Schichten von Foucault. Als der es 1976 eingeführt hatte, war es noch nicht korrekt, sondern ein produktiver Vorschlag (Foucault 1983, V. Kap.).

Neu scheint mir in diesem Buch von Derrida zu sein, dass sich Menschen durch einen Drehspiegel hindurch als von Tieren beobachtet vorstellen sollen, wodurch Jaques Lacans Spiegelstadium problematisiert werde (Derrida 2010, 82-84). - Aber wer dächte heute, nach der Entdeckung der Spiegelneuronen an Makaken und dem allgemein anerkannten Unterschied zwischen Mimikry und Imitation in der vergleichenden Verhaltensforschung, noch mit Lacan, dass die Selbsterkennung im Spiegel das anthropozentrische Privileg des Menschen sei (Lacan 1985, 317ff.)? Auch Menschenaffen, Elefanten und hoch entwickelte Meeressäuger können sich im Spiegel erkennen. Es fragt sich nur, was, wie und wen sie da auf welchem Niveau erkennen, ihr leibliches oder ihr geistiges Ich (siehe im vorliegenden Band 3. Kapitel). Plessner hatte zwischen Mitmachen, Nachmachen und Nachahmung (im Sinne der Darstellung eines Sachverhaltes oder einer Personenrolle) unterschieden. Dem entspricht in der heutigen erfahrungswissenschaftlichen Diskussion die Differenz zwischen dem, was Spiegelneuronen als neurophysisches Korrelat und Mimikry als psychisches Korrelat im Verhalten leisten, sich aber von Imitation unterscheidet (Krüger 2010, 33-36, 151-154; siehe im vorliegenden Band 8. Kap.).

Nun, jeder Verhaltensforscher, der einmal in der Wildnis war, weiß darüber besser zu berichten, wie es ist, wenn einen Menschenaffen, Großkatzen, Skorpione, Giftschlangen und Pythons beobachten, fliehen oder attackieren. In Derridas Schrift bleibt es bei der Hauskatze, die ihn anblickt und die er anblickt, während er nackt von der Dusche kommt, ein schöner Aufhänger, aber für was? - Mir scheint, Derridas Dekonstruktion hatte in seiner Grammatologie und in den Antinomien seiner Gesetzeskraft bessere Tage erlebt.

Agamben geht äußerlich weiter als Derrida, wenn er zunächst die Trennung statt der Vereinigung von Körper und Seele radikalisiert und am Ende eine Exteriorität fordert, ,die weiter außen liegt als jedes Offene, und innen in einer Intimität, die weiter innen liegt als jedes Verschlossene. Das Tier sein lassen, bedeutet also: es außerhalb des Seins lassen“ (Agamben 2003, 100). Aber ist es für die verbliebenen Tiere auf der Welt in Zukunft wirklich besser, sie in der Zone der Nichterkenntnis sein zu lassen? Kann man ihnen so angesichts der Zerstörung ihrer Umwelten durch Menschen helfen? Oder handelt es sich um eine falsche Liberalität, die sie scheinbar ihrer Freiheit überlässt, in Wahrheit aber in ihr sicheres Aussterben stößt? Agambens Radikalität in der Bearbeitung des Tier-Mensch-Themas endet in einem schönen „Shabat sowohl des Tieres 
als auch des Menschen“ (ebd.), der an die Ausstellung von Naturreligionen erinnert, die im Zeitalter des globalen Massentourismus kaum mehr authentisch wirken dürfte. Ist dieser Shabat wirklich die angemessene Antwort auf Nietzsches Frage nach einem postchristlichen Heidentum?

Auffällig ist in beiden Schriften, der von Derrida und der von Agamben, dass beide Autoren von vornherein es nicht für nötig halten, so wie Heidegger in seiner Vorlesung zu verfahren. Heidegger las immerhin noch die seinerzeit führenden Philosophischen Anthropologen (Scheler, Plessner) und theoretischen Biologen wie z. B. von Uexküll, J. Buytendijk, H. Driesch, um seine eigene Umstellung der Frage nach dem Menschen herauszuarbeiten. Derrida und Agamben kennen nichts aus den heutigen Lebens- und insbesondere Verhaltenswissenschaften zum Thema. Sie nehmen auch keine philosophischen Auseinandersetzungen mit diesen Lebenswissenschaften zur Kenntnis, um eine philosophische Kritik anthropologischer Unterscheidungen zu leisten. Längst hat Frans de Waal Schelers Empathiekonzeption in der aktuellen Erforschung von Menschenaffen in ihrem wild life fruchtbar gemacht (de Wal 2008). Schon wieder geht es um den Unterschied und den Zusammenhang zwischen zentrischer und exzentrischer Positionalität in der heutigen Hirn- und Verhaltensforschung, die nicht nur interkulturell, sondern auch interspeziesell vergleichend ins Offene hinein verfährt. Agamben und Derrida genügen indessen die innerhermeneutische Auseinandersetzung mit Heidegger.

Während Sloterdijk Heideggers Vorlesung der philosophischen und historischen Anthropologie zurechnet, weil er Scheler und Plessner kennt, verstehen Derrida und Agamben Heideggers Vorlesung nicht, weil sie nicht wissen, worauf Heidegger antwortet, eben auf Schelers und Plessners Philosophische Anthropologien, von den theoretischen Biologien ganz zu schweigen. Agamben und Derrida verbleiben leider in dem alten dualistischen Vorurteil der Geisteswissenschaft über die Naturwissenschaft. Dazwischen darf es nichts geben, dann wären sie selbst überflüssig. Sie nehmen die inter-biotisch, inter-sozial und inter-kulturell vergleichenden Lebenswissenschaften, deren Ringen um die neuen Erklärungs- und Verstehensaufgaben, besser nicht zur Kenntnis, tun aber so, als ob sie etwas zu deren Thema zu sagen hätten.

Ist es angesichts dieses aktuellen Streites über Heideggers Vorlesung nicht endlich einmal sinnvoll, den Diskurs auf Heideggers Kontrahenten hin zu einem Vergleich auszuweiten?: So wichtig für Agamben die dichterisch nette Zecke (Agamben 2003, 54ff.) ist, als kulminierte ausgerechnet in ihr das Biotische zum Streit um den Menschen, und so entscheidend auch Derrida Heideggers Einebnung der Differenz zwischen Umwelt und Welt vorkommt, er versteht sie nicht. Es ist doch auch heute wieder der Unterschied und die Gemeinsamkeit zwi- 
schen Menschen und Schimpansen aufregender (siehe im vorliegenden Buch 8. Kap.), wie dies bereits für Wolfgang Köhler während des ersten Weltkrieges auf Teneriffa in seinen Schimpansenversuchen der Fall war, an die Lacans Anthropozentrik durch voreilige Schlüsse anschloss. Warum hat Derrida so lange auf Lacan vertraut, statt Schelers und Plessners andere Interpretationen von Köhlers Schimpansenversuchen einmal zu lesen? Da Derrida davon überzeugt war, dass kein großer Philosoph von Platon bis Heidegger auf die „Dummheit“ verzichtet hätte, die Tiere für eine homogene Ganzheit zu halten, wo es doch darum gehe, ihre monströse Hybridizität (Derrida 2010, 69-71) zu entdecken, hätte er gut daran getan, vielleicht doch mal die Pluralisierung der Umwelten und Welten in der philosophischen Diskussion der theoretischen Biologie von von Uexküll im vorigen Jahrhundert zur Kenntnis zu nehmen (siehe im vorliegenden Band 7. Kap.).

\subsection{Humanismus-Kritik durch kopernikanische Dezentrierung des personalen Lebens oder durch exzentrische Anhebung der Lebensmitte in personalen Lebensformen?}

Die Rhetorik, die Größe des Abendlandes aus der umso größeren Größe der eigenen Kritik an ihm zu überblicken, übernimmt Derrida von Heidegger, auch den Gestus der Destruktion. Alle Anderen können so nur klein und da unten sein, nie auf Augenhöhe nicht einmal der Hauskatze. - Plessner hielt dagegen den Wettlauf um die neueste Dezentrierung, d. h. die Permanenzerklärung der Kopernikanischen Revolution, für den ideologischen Kern der westlichen Moderne, ihre ständige Rhetorik der Selbstermächtigung und Selbstüberbietung, die im 20. Jahrhundert zum Hochkapitalismus bestens passe (Plessner 1982e, 97-100; siehe im vorliegenden Band 18. Kap.). Aus Kants Kopernikanischer Revolution werde im 19. Jahrhundert ein Modell zur Verallgemeinerung des gegenseitigen Ideologieverdachts, das sich in der verspäteten Nation der Deutschen im 20. Jahrhundert extremistisch im allgemeinen Autoritätsverfall entlädt, bis nackte Gewalt entscheidet. Niemand behaupte mehr, Dezentrierungen könnten nicht ideologisch verwendet werden, Kopernikanische Revolutionen könnten sich nicht in ihren Folgen gegen ihre anfänglichen Intentionen verkehren! Das Spiel, sich im Namen der nächsten dezentrierenden Revolution an die Spitze der Bewegung zu stellen, alle zu überholen, ohne sie je eingeholt zu haben, war bereits in den 1930er Jahren politisch nicht mehr unschuldig: Die Vernunft wurde im 19 Jh. vom Standpunkt der Gesellschaft entlarvt und entpuppte 
sich so als eine Verschleierung der Klassenzugehörigkeit. Die moderne Gesellschaft wurde vom Standpunkt der Geschichte demaskiert, in der jede Epoche gleich nah zu ihrem jeweiligen Gotte war. Alles wurde relativ und kontingent, bis auf den absoluten Standpunkt der Relativität und Kontingenz selber. Gesellschaft und Geschichte wurden vom Standpunkt der Darwinschen Revolution aus dezentriert. Die Gesellschaft tauchte so als Vitalisierung in der Geschichte auf und erstarrte wie Pompej in der Asche des Vesuvs erstorben war. Schließlich wurde das biologisch übrig gebliebene Leben vom Standpunkt der Rasse demaskiert, und die Folgen kennen wir. Immer wurde in diesem intellektuellen Wettlauf um die neueste Dezentrierung mit dem Anspruch einer erneuten kopernikanischen Revolution eine Teilerkenntnis auf das Wesen des Menschen im Ganzen übertragen und damit zu einer Ideologie, d. h. zu einem Bewusstsein, das standpunktgebunden notwendiger Weise als falsch entlarvt wurde. Wer sich gegenseitig ressentimentgeladen auszieht, ohne ein privates Verhältnis miteinander zu haben, landet so in öffentlichen Auseinandersetzungen um das Politische früher oder später im Bürgerkrieg.

Das Modell der Kopernikanischen Revolution, natürlich gegen die Intention in Kants Agnostizismus wider jeden Fanatismus praktiziert, setzte eine Serie von Entlarvungen und Demaskierungen in Gang, die politisch immer neue Kollektive zur Selbstermächtigung gegen die alten Autoritäten legitimierte, bis die Zivilisation abgeschafft war: „Statt den Menschen von jeder Autorität weg- und zu dem Zentrum seiner ursprünglichen Entscheidung hinzuführen, verdeckt sie [die Logik der Aufklärung des Bewusstseins: HPK] diese Ursprungsmitte mit immer neuen Autoritäten, deren Basis dem naturhaften Sein ständig enger angenähert wird“ (Plessner 1982e, 132). Dieses Problem war kein allein spezifisch deutsches Problem, es hat sich inzwischen globalisiert, worüber jede Dekonstruktion des Humanen als Metaphysik und Onto-Theologie im Anschluss an Heidegger, zumal zu Beginn des 21. Jahrhunderts, nachdenklich werden sollte:

Das 19. Jahrhundert hatte den Unglauben an Gott der Öffentlichkeit zum Bewusstsein gebracht, ihren Glauben an den Menschen aber noch nicht zu erschüttern vermocht. Das 20. Jahrhundert hat sogar noch diesen Glauben, den Humanismus im öffentlichen Bewusstsein getötet und das Leben ohne jede metaphysische, geschichtliche oder natürliche Autorität und Verheißung nicht nur unausweichlich gemacht, sondern zum praktischen, ja politischen Postulat erhoben. Nur dieses Maß der Entgötterung und Entmenschung macht es begreiflich, dass gerade hochzivilisierte Nationen zur Selbsthilfe einer künstlichen autoritären Bindung im Politischen greifen, um die elementaren Daseinsinstinkte vor den nihilistischen und defätistischen Schlussfolgerungen der Intelligenz zu schützen. (Plessner 1982e, 114) 
Wenn Plessner vom Verlust der Verhaltensmitte in personalen Lebensformen des Säkularisierungsprozess (von der Renaissance bis zum Beginn des 20. Jahrhunderts) spricht, geht es nicht um die Wiederherstellung der christlichen Achse, um welche sich das Welt- und Selbstverständnis personalen Lebens zuvor drehen konnte (Plessner 1982e, 93f., 98f., 222f.). Umgekehrt: Was rückt denn als lebendige Verhaltensmitte für Personen vor, wenn sich der Humanismus nach einer Serie kopernikanischer Dezentrierungen seit dem Beginn des 20. Jahrhunderts erschöpft hat? Was tritt künftig an seine „Stelle im Funktionszusammenhang des Lebens“ (ebd., 223), den es schon vor der westlichen Moderne gegeben hat und der auch noch nach ihr nötig sein wird. Was fungiert wie an seiner Stelle in einer neuen Achsendrehung, die als dritte den beiden vorangegangenen Achsendrehungen, eben der Entstehung der Achsenkulturen (darunter der Monotheismen) und der Verweltlichung des Christentums, folgen dürfte? Kann der bislang nachrückende Dezisionismus, der keine Prinzipien mehr über die Situation hinaus kennt, also bewegungspolitisch in die schiere Selbsterhaltung von Situation zu Situation führt, diese Mitte personaler Verhaltensbildung in der Generationenfolge ermöglichen? Wohl kaum auf Dauer, nur bis die Ressourcen des Christentums und des Humanismus verbraucht sein werden:

Die normlos gewordene Entscheidung hat nichts mehr über sich, sondern nur noch etwas vor sich: eine konkrete Lage, die gemeistert sein will. Und sie hat hinter sich keine allgemeinen Rückgriffsmöglichkeiten und Rechtfertigungen aus abstrakten Idealen mehr, sondern nur noch eine massive Realität: das Volk und seinen Selbsterhaltungstrieb. (Ebd., 168)

Plessner geht es (schon seit 1924) strukturfunktional betrachtet um die Ermöglichung einer zivilen Gesellschaft für lebende Personen, die sich in private und öffentliche Personen verdoppeln dürfen, um mit ihrer brüchigen Natur und Kultur in ihrer hybriden Lebensführung auskommen zu können. Er missversteht nicht die bürgerliche Gesellschaft als die allein kapitalistische Gesellschaft, wie dies sowohl linke als auch rechte Gesellschafts- und Kulturkritiker im Namen ihrer jeweiligen Gemeinschaftsideale taten. Er dachte nicht partei- und bewegungspolitisch, sondern strukturell und funktional im Hinblick auf eine gemeinsam zu teilende Welt personaler Lebensformen. Sie fungiert in Öffentlichkeiten, in denen sich einander Unbekannte, Andere und Fremde als Personen ohne Assimilationszwang begegnen können, also einander unbekannt, anders und fremd bleiben dürfen, nicht müssen (Plessner 1981b, 94-96, 102f., 133). Sie können dies, indem sie sich diplomatisch und taktvoll zueinander verhalten (ebd., 97f., 105f.), weil sich die wirkliche Pluralität ihrer grundlegenden Werte nicht anders als konfliktuell am Rande der Gewalt ausleben lässt. Diese öffentli- 
che Zivilisierung der Gesellschaft ermögliche Formen der Individualisierung, die in den linken und rechten Vergemeinschaftungen der Individuen nur als Entfremdungen vom Gemeinschaftswesen des Menschen gedacht werden konnten. Plessners Bejahung der Entfremdung im Wesen des Menschen hatte einen gänzlich anderen Sinn als den der Bejahung einer Totalisierung von Kapitalismus wider alle Gemeinschaftlichkeit. Sie erfolgte um des Persönlichen willen, das der politischen Verschränkung von Gemeinschafts- und Gesellschaftsformen in zivilen Öffentlichkeiten bedarf (ebd., 115f.). Sein Hauptwerk endet in dem Bewusstsein einer im 20. Jahrhundert neu nötigen Achsendrehung, aus der heraus auch die Anfänge des Christentums neu angeeignet werden können: Geist gehe nicht wie der Glaube „immer nach Hause“, sondern zerstöre den Weltkreis und tue „uns wie der Christus des Marcion die selige Fremde auf“ (Plessner 1975, 346).

Wie man bei Plessner lernen kann, ist die Unterscheidung der folgenden Fragen sinnvoll: Wer und was wird dezentriert, von woher und von wem wird dezentriert, und worin bestehen die Folgen dieser oder jener Dezentrierung in der räumlichen und zeitlichen Verteilung von Verhaltensbildungen? Spätestens im Hinblick auf die Folgen hat Plessner die Hypothese entwickelt, dass Kopernikanische Revolutionen, also im heutigen Sprachgebrauch: Dezentrierungen ohne Rezentrierungen im Leben als Prinzip schief gehen, weil sie dieses Leben erneut dualistisch aushebeln zugunsten seiner Beherrschung durch ein Kollektiv abgespaltener Subjekte. Deshalb bleibe ich in seiner Begriffswelt: Exzentrieren bedeutet bei ihm nicht nur, dass sich etwas oder jemand aus sich heraussetzt und von diesem Außen aus positioniert. Exzentrieren bedeutet auch, dass das Herausgesetzte nicht ausschließlich von dort, wo es jetzt ist, leben kann. Es gibt im personalen Leben keine vollständige Exzentrierung, die endgültig ins Reine führen könnte, etwa zu den Engeln, zu Gott, in die Unendlichkeit der Semiose, in das Differieren der Differenzen (Derrida), in die Trennung von Körper und Seele (Agamben), in die jeweils eine Seite unter Ausschluss der jeweils anderen Seite von Dichotomien. Eine Exzentrierung wird durch Rezentrierung lebbar, und diese Lebbarkeit läuft über die Leiblichkeit der betroffenen Teilnehmer in der geistig von ihnen geteilten Mitwelt (siehe Plessner 1975, 302ff.), nicht über die vermeintlich interesselose Reflexion von bloßen Zuschauern oder Betrachtern.

Die Exzentrik stellt die Konzentrik der je eigenen Verhaltensbildung in einen Weltrahmen, in dem jede Person auch da stehen kann, wo die andere Person stehen könnte. Wer als Person exzentrisch lebt, vollzieht ihre Konzentrik angehoben in dem Weltrahmen, nicht nur leiblich eingespielt auf die Umwelt. Die personale Art und Weise zu leben ist nicht einfach vorgegeben, sondern 
aufgegeben. Wer exzentriert, kommt nie endgültig und vollständig dort an, wohin die Exzentrierung ging, denn sie musste auch konzentrisch vollzogen werden. Und wer von dort zurückkommt auf sich, sich also rezentriert, erreicht sich auch nicht bleibend und gänzlich da, wo er schon einmal war. Es geht bei der Exzentrierung und Rezentrierung um eine Zwischenwelt von Vollzügen, in denen verschieden mögliche Bewegungsrichtungen nach geistiger Orientierung hier und jetzt verschränkt werden. Im raum- und zeithaften Wechsel zwischen Exzentrierung und Rezentrierung werden die Brüche, die zwischen den Verhaltungsrichtungen Ex und Re liegen, in einer Verschränkung vollzogen (siehe Plessner 1975, 290; im vorliegenden Buch 3. Kapitel).

Plessners Philosophische Anthropologie steigt also aus guten Gründen aus der Serie kopernikanischer Revolutionen, die sich alle von rechts und von links überbieten wollen, aus. Sie nimmt an dem intellektuellen Überbau des Hochkapitalismus nicht teil, sondern fragt nach der Gewinnung einer dritten Achse von Welt- und Selbstverständnissen, die mit dem Hochkapitalismus abständig umgehen könne und wofür sie mindestens eine dem Diesseits immanente Transzendenz brauchen (siehe im vorliegenden Band 17. Kapitel). Wie kam es demgegenüber für Derrida geschichtlich dazu, Heideggers Humanismus-Kritik durch Dekonstruktion überbieten zu müssen? Wie erschien ihm damals, 1968, exemplarisch für das neue französische Philosophieren in seinem Umkreis, die Problemlage im Anschluss an Nietzsche und Heidegger, deren Philosophien bei ihm für die beiden Fines hominis stehen?

Derrida verstand sich schon seinerzeit als in „dem antihumanistischen und antianthropologischen Gegenstrom“ schwimmend, „in dem wir uns befinden“ (Derrida 1988, 141). Dieser Gegenstrom reagierte auf einen vorangegangenen Strom des „Humanismus oder Anthropologismus“, der „den gemeinsamen Boden der christlichen oder atheistischen Existentialismen“ (ebd., 139) abgegeben hatte. Neben dem christlichen Personalismus waren mit den atheistischen Existentialismen einerseits Jean-Paul Sartres phänomenologische Ontologie (im Anschluss an Husserl, Heidegger und Hegel), die Derrida Heidegger getreu für eine philosophische Anthropologie hält, und andererseits Hegel-Marxismen gemeint, die auf Alexandre Kojève und das Studium des jungen Marx (Entfremdung des Menschen von seinem Wesen in der gemeinschaftlichen Arbeit) zurückgingen. - Kurzum: Es gibt problemgeschichtliche Lagen, in die man aus diesen und jenen Zufällen heraus hineingeboren wird, aber was macht man daraus? Derrida lässt jedes Problembewusstsein über die o. g. Funktion des Christentums und des Humanismus und deren Vakanz in der westlichen Moderne vermissen. Er gibt auch keine Gründe dafür an, warum es besser sein sollte, auf der antihumanistischen als auf der humanistischen Seite zu stehen 
(siehe im vorliegenden Band 19. Kapitel). Die Möglichkeit, diesen ganzen Gegensatz in Frage zu stellen, indem man seine historische Funktion rekonstruiert, taucht überhaupt nicht auf. Dies hatte Foucault in der Ordnung der Dinge (1966), die nicht einmal erwähnt wird, immerhin versucht. Aber auf Grund seiner 1968er Erfahrung nahm er seine frühere Hypothese vom „Ende des Menschen“ in den 1970er Jahren zurück (Foucault 1996, 84f.; siehe Krüger 2009a, 40-53).

Nehmen wir einmal mit Derrida 1968 an, es sei aus historisch kontingenten Gründen (Emanzipation der jungen von der alten Generation, der philosophisch Interessierten von den partei- und bewegungspolitisch Engagierten im Konkurrenzkampf der Intelligenzen) plausibel gewesen, seine Position $\mathrm{zu}$ beziehen, und unterstellen wir weiter mit ihm die französisch hochselektive Rezeptionslage der Werke von Husserl, Heidegger, Nietzsche, Marx, Hegel, vor allem durch Levinas, der Derrida auch keine Erwähnung wert ist, und Kojève vermittelt, dann bestand Derridas ganzer Einsatz darin, Folgendes aufzuzeigen: Hegels, Husserls und Heideggers Philosophien ergeben einerseits ein Kritikpotential an Anthropologismen, verbleiben aber andererseits im Rahmen einer Metaphysik, die „Onto-Theologie“ ist. Dieser Befund ist nicht überraschend, einerseits weil die Transzendentalphilosophie von Anfang an Anthropologie-Kritik war (Kant versus Herder, siehe Krüger 2009a, 105-129) und andererseits weil sich in der modernen Metaphysik die christliche Theologie verweltlichte, zumal in Deutschland: Philosophie nach deutscher Auffassung ist

[...] verweltlichte Theologie, ein vom Schicksal erzwungener grandioser Schadenersatz, Phänomen eines Geistes, der zu aufgeklärt ist, um christlich zu sein, aber nicht aufgeklärt genug, um es wieder zu werden. Die Tatsache, dass das ganze 19. Jahrhundert eben diese ihre ideologische Funktion durchschaut hat, dass es hinter Marx, Kierkegaard und Nietzsche kein Zurück mehr gibt, bestimmt in dem Umkreis einer beruf-spezialistischen Arbeitswelt die gegenwärtige Lage der deutschen Philosophie, damit aber das deutsche Bewusstsein und seine Antwortmöglichkeiten auf die Zeit. (Plessner 1982e, 188)

Insoweit hätte sich Derrida in den 1960er Jahren durch die o. g. Rezeptionen in eine Problemlage aus den 1920er Jahren eingearbeitet. In der Tat enthielt die Metaphysik das doppelte Ende des Menschen, angesichts seiner Sterblichkeit und im Hinblick auf seine Idealisierungen. Mit Gott starb sein Gegensatz und seine Einheit mit dem Menschen. Aber daraus erwuchs nicht nur mit der „Entgötterung“ die „Entmenschung“, sondern auch das Problem der „säkularisierten Vergottung des Menschen“ (Plessner 1981d, 150, 221), für das sich bei Derrida keine Anhaltspunkte, etwa im Sinne der o.g. dritten Achsenverlagerung, ergeben.

Stattdessen sympathisiert Derrida innerhalb des vorgegebenen Rahmens mehr mit Nietzsche als mit Heidegger. Heideggers Philosophieren leide am 
Magnetismus der Eigentlichkeit, eine sehr treffende Beschreibung der Sogwirkung seiner Texte, an einer ontischen Nähe, die auf dem ontologischen Wege der Entfernung vom Sein doch wieder in die Selbstpräsenz und deren Nachbarschaft münde (Derrida 1988, 150-153). Derrida wollte über Heideggers Art und Weise von Dekonstruktion, „durch die Wiederholung des Impliziten“ gegen eben dieses Gebäude anzugehen, hinausgelangen „in einen Wechsel des Standortes“, „auf diskontinuierliche und plötzliche Weise, durch ein brutales Sichaußen-Einrichten und durch die Affirmation absoluten Bruches und absoluter Differenz“ (ebd., 155f.). „Kein Zweifel, dass Nietzsche zum aktiven Vergessen des Seins gemahnt hat; es hätte nicht die metaphysische Form gehabt, die Heidegger ihm aufgebürdet hat" (ebd., 157).

Plessner hatte demgegenüber Derridas Rahmen, zwischen Heidegger und Nietzsche eine Wette aufs Philosophieren einzugehen, den auch Foucault philosophisch teilte, aufgekündigt. Versteht man Nietzsches Nihilismus richtig, d. h. gerade nicht als Dezisionismus, sondern als die o. g. Frage nach einer exzentrisch angehobenen Funktionsmitte in den personalen Lebensformen nach der westlichen Moderne und ihrem Humanismus, dann gilt es, als neuen Rahmen die Welt ohne ihren Schöpfungscharakter zu denken. Durch das Schöpfen zieht sich zwar eine Art Senkung von Gott über den Menschen bis zum Dezisionisten der Situation hindurch, aber vor allem eine Kontinuität des eigenen Selbst in seiner nächsten Nähe noch auf allen Umwegen. Ein philosophisch durchdachter Atheismus Nietzsches, der mehr sein soll als bloßer A-Theismus, verlangt einen höheren Einsatz in einer Art von Pascalscher Wette:

Der Mensch kann sich und damit die Welt, das Sein des Seins, noch höher verstehen, nicht hermeneutisch, und vielleicht ist die oben angedeutete Aufrollung einer Position zwischen Ernst und Nichternst, Notwendigkeit und Nichtnotwendigkeit, die zum ,Seinsproblem', zur Welt als dem Nichtseienden, Nichtlebenden, Nichtwesenden und nur in einzelnen Konkretionen Seienden, Lebenden, Wesenden konforme Haltung. Die Welt als das Nichtkreatürliche, Unschöpfbare - Unvergängliche und doch nicht Ewige (auch nicht ewig, insofern es zeitigt). Das erst wäre Atheismus. (Plessner an König am 22. 2. 1928 in König/Plessner 1994, 181)

„Mensch-Sein ist das andere seiner selbst Sein“ (Plessner 1981d, 225). Wie hat es im Frankreich der 1960er Jahre dazu kommen können, sich die Agenda des Philosophierens durch Heidegger und Nietzsche vorgeben zu lassen? Schienen Hegel und Marx zu sehr partei- und bewegungspolitisch okkupiert worden zu sein? Wie hat man Heideggers Kritik an der philosophischen Anthropologie glauben können und dementsprechend das Thema einer Philosophischen Anthropologie (im Unterschied zur anthropologischen Philosophie: Plessner 1983d, 36; Plessner 1983i, 243) mit Sartres phänomenologischer Ontologie für erschöpft 
halten können? Derrida selbst spricht von anthropologischen Schemata, denen aber nur die anderen gefolgt sein sollen. Galt Scheler in Derridas Kreisen einfach als zu katholisch, weshalb man ihn nicht ernst nehmen konnte? Gab es unterschwellig für Derrida in Husserl eine versteckte Hoffnung darauf, als Jude führend angekommen zu sein in der westlichen Moderne, eine Hoffnung, die man dann aber mit Husserls tragischem Scheitern an Heidegger als Hermeneutiker umso umwegiger mit und gegen Heidegger erfüllen musste? Spielte hier also Hannah Arendts Unterscheidung zwischen Paria und Parvenu eine Rolle (Arendt 1981a)? Wirkte die alte französische Attraktion Rousseau auf neue Weise, d. h. im ethnologischen Strukturalismus und eben magnetisch in der Heidegger-Rezeption, nun jedoch so penetrant, dass endlich ein Befreiungsschlag in die Ferne nötig wurde? Und entlud sich das ganze französische Selbstbewusstsein dann in dem Ausbau der Umwege von de Saussure aus in eine Grammatologie und Archäologie, um den eigenen Strukturalismus dezentrieren zu können? - Gewiss folgten aus diesem vorgegebenen, aufgenommenen und umgebauten Rahmen originelle Beiträge wie die Derridas, Foucaults und vor allem von Deleuze, was eine Naturphilosophie angeht, aber diese Frage nach dem „Wir“, die Derrida selbst stellte, unterliegt einer starken historischen Kontingenz, die systematisch nicht überzeugt. Auf jeden Fall lohnte sich die Untersuchung, wie es zu dieser Schließung eines Man im Wir gegen die Öffnung der Philosophischen Anthropologie hat kommen können.

Hat sich die Dekonstruktion, als Fortsetzung der Heideggerschen Humanismuskritik, ernsthaft darüber Rechenschaft gegeben, was ihre Überbietung noch Heideggers an Autoritärem in der Biopolitik und an Verwertbarem im globalen Hochkapitalismus befördert hat? Was soll an dieser Affirmation des Anti-Humanismus so radikal oder fundamental kritisch sein, wenn sie keine produktive Alternative im Welt- und Selbstverständnis personalen Lebens zustande bringt? Gehört dieser kritische Gestus mit seinen affirmativen Auswirkungen nicht zu dem, was Plessner die politische Verführbarkeit des bürgerlichen Geistes nennt, gleich im Untertitel seiner Verspäteten Nation?

\subsection{Noch einmal Heideggers anthropozentrische Dezentrierung dezentrieren oder Plessners natur- und geschichtsphilosophische Kritik an Heideggers Zentrismus ernst nehmen?}

Da Agamben und Derrida nicht wissen, wovon sich Heidegger in seiner Vorlesung abzugrenzen versucht hat, komme ich exemplarisch auf Plessners Philo- 
sophische Anthropologie zurück, um zum Vergleich beider quasitranszendentaler Unternehmungen in der künftigen Diskussion einzuladen. Dafür gibt Heidegger den strittigen Punkt selbst vor, an dem sich auch Derrida und Agamben reiben. Er ebnet Schelers und Plessners Unterscheidung zwischen Umwelt und Welt ein, wenn er nur noch von den oben zitierten weltlosen Steinen, den weltarmen Tieren und den weltbildenden Menschen (Heidegger 1983, 263) spricht. Für Agamben und Derrida ist diese Unterscheidung nur eine Differenz unter vielen, die Heideggers Anthropozentrik zeige und die es im Namen einer transzendierenden Exteriorität/Interiorität (Agamben) oder absoluten Differenz (Derrida) zu überschreiten gelte. Die Auflösung der Unterscheidung in eine Vielzahl von graduellen Übergangsdifferenzen soll dichotomische Aussagen über den vermeintlich einen Wesensgegensatz zwischen Mensch und Tier abwehren. Dies ist moralisch gut gemeint, um den Wesensgegensatz nicht als Legitimationsgrund für die Herrschaft des Menschen über Tiere zu missbrauchen, aber verantwortungsethisch kurzsichtig, weil Menschen in der ökologischen Krise Tieren nicht helfen können, ohne den Wesensunterschied ihrer Lebensformen in ihren nicht-intendierten Handlungsfolgen zu berücksichtigen. Der philosophische Grund für die Einebnung der Differenz zwischen Umwelt und Welt war bei Heidegger auch ein ganz anderer: Der existenziale Bezug auf den Sinn von Sein im Selbstverständnis des Daseins soll ontologisch fundamentaler sein als diese Welt-Umwelt-Differenz, also als die vermeintliche Regionalontologie der philosophischen Anthropologie. Überprüft man Heideggers Karikatur von der Philosophischen Anthropologie, trifft man in letzterer auf eine Kritik an Heideggers Anthropozentrik, von der Heidegger durch seine Karikatur offenbar ablenken wollte, worauf Agamben und Derrida hereingefallen sind.

Plessner hat in der Tat Heideggers Sein und Zeit als eine Anthropozentrik kritisiert, aber nicht nur lange vor, sondern auch anders als Agamben und Derrida. In seiner Naturphilosophie, die den Ermöglichungsgrund anthropologischer Vergleiche zwischen personalen und non-personalen Lebensformen vertikal rekonstruiert (siehe im vorliegenden Buch Erster Teil), wirft Plessner Heidegger vor, die philosophische Tradition des Subjektivismus fortzuführen, nun in der Gestalt von Existenz. Heidegger meine, „dass der Untersuchung außermenschlichen Seins eine Existentialanalytik des Menschen notwendig vorhergehen müsse“, weil er glaube, dass der „philosophisch Fragende sich selbst existentiell der Nächste und darum der sich im Blick auf das Erfragte Liegende“ (Plessner 1975, V) sei. Dieser existenzialen Zentrik von Selbstnähe stellt Plessner die Exzentrizität der personalen Lebensform gegenüber, die nicht nur vom Nächsten auch zum Fernsten befreie, sondern ermögliche, „sich weder der Nächste noch der Fernste zu sein“ (ebd.). Der Mensch könne „trotz des 
nichtseinsmäßigen Charakters seiner Existenz in eine Reihe mit allen Dingen dieser Welt“ gehören (ebd., V-VI).

Schelers Ekstasis kehrt hier in transformierter Gestalt naturphilosophisch wieder. Die belebte Natur ist bei Plessner kein Herrschaftsobjekt, sondern ein „Subjekt-Objekt“ (Plessner 1975, 32), das von sich aus seine Grenzübergänge vom Organismus zur Umwelt realisiert (ebd. 103f.). Anderes und fremdes Leben kann man sich selber zeigen lassen, wenn man ihm den ihm nötigen Spielraum und die ihm nötige Spielzeit einräumt. Plessner deckt hier kategorial reale Möglichkeiten des Lebenkönnens von Körpern auf, die sich von den empirischen Begriffen der Biologie unterscheiden. Er vollzieht eine Wende der transzendentalen Frage nach der Ermöglichungsstruktur der Erfahrung vom abgespaltenen Subjekt der Erkenntnis weg in den Gegenstand der forschungspraktischen Erfahrung selbst hinein, insofern dieser Gegenstand von selbst lebt, wofür man ihm seine Verhaltensfreiheit zu gewähren hat. Darin besteht ein Muster für die quasi transzendentale Art und Weise zu philosophieren: Insoweit die Ermöglichungsfrage weiterhin gestellt wird, handelt es sich um eine transzendentale Unternehmung. Aber diese Frage wird von der bewusstseinsphilosophischen Annahme, der Gegenstand sei nur als von einem Subjekt konstituiertes Objekt möglich, klar emanzipiert. Dies erlaubt es, dem Subjekt-Zirkel im Selbstbewusstsein zu entgehen. Was in den Forschungspraktiken real und symbolisch getan wird, und zwar von Körpern, die selber leben, indem sie miteinander interagieren, befreit sich im Maße seiner Reproduzierbarkeit von dem, was einzelne Bewusstseinssubjekte bewusst leisten können. Daher lässt sich das transzendentale Rückschlussverfahren auf das Sich-Positionieren von Lebewesen unter Lebewesen umlenken, statt es in das Bewusstsein vom Bewusstsein zu verlegen, statt also in reflexionsphilosophische Zirkel zu geraten. Die Ermöglichungsstrukturen von Lebenserfahrung werden in denjenigen Lebensprozessen entdeckt, an denen die Untersuchten und die Untersucher als selber Lebewesen in den verschiedensten Lebensformen (azentrisch, konzentrisch, exzentrisch) teilnehmen (siehe im vorliegenden Buch 1. und 3. Kapitel).

Daher kritisiert Plessner ausdrücklich das Streben nach einer alten Metaphysik im Sinne der strengen Wissenschaft, das von Kant bis Husserl und Heideggers Sein und Zeit in Analogie zu Kants Kritik der reinen Vernunft verfahren wolle. Es folge zwei stillschweigenden Präsuppositionen, nämlich der Frage nach dem Sinn von Sein, die der ersten Achsendrehung zum Christentum entsprochen habe, und dem neuzeitlichen Primat der Erkenntnistheorie über die Philosophie, das die kopernikanische Revolution als die zweite Achsendrehung in die Säkularisierung des Christentums zum Ausdruck bringe (zu den Achsenverlagerungen im vorliegenden Band 17. Kapitel). Das Hauptproblem einer der- 
art reflexionsphilosophisch konzipierten Metaphysik bestehe dann darin, dass sie keine Grenze mehr angeben könne, da das Bewusstsein schon immer bei den Gegenständen sei und die Reflexion bereits im Bewusstsein ansetze. Die Gegenstände außerhalb des Bewusstseins und die Reflexion innerhalb des Bewusstseins verfestigen sich zu zwei getrennten Seinsbereichen, deren Grenzübergang ineinander in der Luft hänge, statt in einer exzentrischen Positionalität verschränkt werden zu können (siehe Plessner 2002, 32-63). Der Dualismus entgrenzt den Herrschaftsanspruch durch eine Serie kopernikanischer Revolutionen hindurch auf alles Machbare, statt umgekehrt den Herrschaftsanspruch über die Natur und sich selbst für die Lebensmitte personaler Lebensformen inmitten der Natur zu begrenzen.

Plessner kritisiert Heideggers Vorgehen aber nicht nur naturphilosophisch, sondern auch geschichtsphilosophisch im Hinblick auf den horizontalen Vergleich verschiedener Welt- und Selbstverständnisse in den personalen Lebensformen der Zivilisations- und Weltgeschichte als eine Anthropozentrik. Plessner schreibt in Macht und menschliche Natur (1931):

Fraglos muss eine Analyse des Wesens Mensch, die so angesetzt ist wie die Heideggersche, in der einheimischen Region dessen, was ich je selbst bin, anfangen. Und auf ihrem Wege werden ihr die typischen Lebensvollzüge auftauchen, die unser Dasein, das Dasein von Europäern, die von klassisch-christlicher Tradition geformt sind, nun einmal beherrschen. Ausgerichtet auf eine Strukturformel, die mit der Auslegung des eigenen auch die mögliche Auslegung fremden Daseins verbindend alles Menschliche der Möglichkeit nach oder dem Wesen nach auf seine Essenz und sein Specificum hin ausdrücken soll, kann dieser Weg, der schließlich in der Formulierung des Sinns von Sein schlechthin enden will, sich sehr wohl als bloßer Weg ausgeben. Die Existentialanalyse mit den Stationen der Sorge, des Seins zum Tode, der Angst hat immer die Möglichkeit, den jeweiligen Gehalt auf die Methode zu relativieren und zu sagen: das ist unser Zugang zum menschlichen Wesen; nur auf jenem Lebensboden, dem die Fraglichkeit dessen, was er eigentlich ist, zugehört, kann Wesensanalyse genuin getrieben werden. (Plessner 1981d, 157)

Freilich verträgt sich diese methodische Relativität nicht mit Heideggers Anspruch auf eine Fundamentalontologie. Daher fragt Plessner weiter, auch angesichts von Heideggers Buch Kant und das Problem der Metaphysik (1929), ob die ,anderen Auslegungsformen der Existenz“ mit den eigenen Auslegungsformen „nicht nur gleich möglich“, „sondern auch gleichberechtigt“ seien (Plessner 1981d, 157).

Für Plessner kann Heideggers Existenzphilosophie andere Auslegungsformen der formalen Struktur von Existenz nicht als gleichberechtigte behandeln:

Die Bedingungen der Möglichkeit, Existenz als Existenz anzusprechen, haben zugleich den Sinn, Bedingungen der ,Möglichkeit‘ zu sein, Existenz als Existenz zu führen. Eine Vor- 
zugsstellung der Kultur, in der diese Möglichkeit faktisch (oder nur möglicherweise faktisch) ist, vor anderen Kulturen, welche diese Möglichkeit von sich aus nicht haben, ist damit gegeben. (Plessner 1981d, 158)

Daraus folgten zwei Varianten, sich gegenüber diesen anderen Kulturen zu verhalten: Entweder wolle man sie dann zur Umkehr zu sich führen wie die Christen die Heiden, oder man spreche ihnen das Menschsein ab, denn sie leiden am Maßstab der Existenzialität gemessen an einem „eminenten ,Mangel` an Individualität, Personalität und Freiheit zu Möglichkeiten“, an der „,Verfallenheit an das Man““ (ebd., 159).

Gegen Heideggers Existenzialität als anthropologischen Maßstab für geschichtliche Vergleiche erhebt Plessner zwei Einwände. Würde man erstens Heideggers Orientierung auf das je eigene Verständnis konsequent befolgen, dann müsste man auch das je Eigene der anderen Auslegungen respektieren, mithin es dem anderen Menschen überlassen und gewaltlos von ihm erfahren,

[...] was er ist und als was er sich auslegt, ob er die Essenz seiner Existenz in seine Existenz oder: einen anderen Menschen, in Tier oder Pflanze, in Stern oder Erde, in einen Gott oder in die Elemente setzt. Die vom Abendland gewonnene Weite des Blicks erfordert die Relativierung der eigenen Position gegen die anderen Positionen. Ihr Mittel und ihre Entdeckung ist der Begriff Mensch, sind im Grunde alle formalen oder formalisierbaren Kategorien wie Leben, Kultur, Welt. (Plessner 1981d, 159)

Dieser Forderung trägt Plessner dadurch Rechnung, dass er seine eigene philosophische Unternehmung am Prinzip der Unergründlichkeit des Menschen, d. h. an der Offenheit geisteswissenschaftlicher Fragen, als Erkenntnisprinzip und Erkenntnispraxis ausrichtet (siehe im vorliegenden Band 16. Kapitel).

Plessners zweiter Einwand gegen Heideggers Maßstab der Existenzialität betrifft den formal apriorischen Charakter der Methode. Heidegger verteidigt seine eigene Daseins- und Existenzialanalyse immer wieder, indem er ihren gegenüber materialen Inhalten neutralen Charakter von Strukturen hervorhebt, so auch in Kant und das Problem der Metaphysik, eine Selbstauskunft, die Plessner ernst nimmt: „Denn die formal-dynamische Struktur der Existenz gestattet ihre Abwandlung in die verschiedensten faktisch gewordenen Existenztypen oder Typen des ,In der Welt Seins““ (Plessner 1981d, 156). Demgegenüber hält Plessner nicht nur an Schelers auch material-apriorischer Verfahrensweise fest, sondern fordert darüber noch hinausgehend eine neue „Verbindung apriorischer und empirischer Betrachtung nach dem Prinzip der Unergründlichkeit des Menschen“ (ebd., 160). Für die geschichtliche Erfahrung sei gerade der Wechsel zwischen dem Ermöglichenden und dem Verwirklichten durch Vergegenwärtigung des Künftigen charakteristisch. Wenn man nicht nur formale 
Funktionsstrukturen, sondern auch deren materiale Inhalte berücksichtigt und zudem in der spezifisch geschichtlichen Erfahrung den Wechsel zwischen ihrer Ermöglichung und dem in ihr Verwirklichten veranschlagt, dann führt dies zu einer Diagnose der weltgeschichtlichen Lage. Für Plessner löst sich der Westen vom Westen in der Globalisierung des Hochkapitalismus ab, wodurch alle soziokulturellen Welt- und Selbstverständnisse fraglich werden. Das Spektrum der Antworten auf diese säkulare Fraglichkeit des Menschen reicht von Diktaturen bis zu demokratisch-gewaltenteiligen Verfahren, was geistig-kulturell von der Modernisierung verschiedener Achsenkulturen $\mathrm{zu}$ auch nicht-westlichen Modernen abhängt. In dieser Offenheit einer zivilisatonsgeschichtlichen Zukunft zwischen der veränderten westlichen Moderne und den nicht-westlichen Modernen liegen sowohl Gefahren als auch Chancen, zwischen denen man differenzieren kann. Personale Lebensformen können als solche nicht ohne einen utopischen Standort existieren, der zumindest eine ihrer Welt immanente Transzendenz darstellt (siehe im vorliegenden Band 17. Kapitel).

Weder Agamben noch Derrida haben naturphilosophisch und geschichtsphilosophisch vergleichbare Kritiken an Heideggers Anthropozentrik zustande gebracht. In Plessners exzentrischer Positionalität und seinem homo absconditus leben Figuren der Ekstasis fort, aber auf eine transformierte und verhalten indirekte Weise. Schelers Ekstasis war wohl am Ende für Heidegger doch nur ein verführerisches Maskenspiel, wenn man seine eigene, sorgfältig geplante Demaskierung in den Schwarzen Heften bedenkt. Hannah Arendt hatte sich über Heideggers Philosophie nicht täuschen lassen, als sie 1946 über seine Existenzauffassung schrieb: „Der wesentliche Charakter dieses Selbst ist seine absolute Selbstischkeit, seine radikale Abtrennung von allen, die seinesgleichen sind“ (Arendt 1990, 37). Heidegger habe später versucht, „,seinen isolierten Selbsten in mythologisierenden Unbegriffen wie Volk und Erde wieder eine gemeinsame Grundlage nachträglich unterzuschieben“ (ebd., 38). Dies diene dazu, „die nur sich wollenden Selbste in einem Überselbst zu organisieren, um die in der Entschlossenheit ergriffene grundsätzliche Schuld irgendwie in die Praxis überzuleiten“ (ebd.). Es sei evident, „dass derartige Konzeptionen nur aus Philosophie heraus- und in irgend einen naturalistischen Aberglauben hineinführen können“ (ebd.). 